\title{
CLASSIFICATION OF SKEW SYMMETRIC MATRICES
}

\author{
BERNDT BRENKEN
}

(Communicated by Palle E. T. Jorgensen)

\begin{abstract}
The group $\operatorname{GL}(d, \mathbb{Z})=\operatorname{Aut}\left(\mathbb{Z}^{d}\right)$ acts on the $\mathbb{Z}$-module $\operatorname{Hom}\left(\Lambda^{2} \mathbb{Z}^{d}\right.$, $\mathbb{Z} / a \mathbb{Z})$ by $\varphi \rightarrow \varphi(\alpha \wedge \alpha) \quad\left(\alpha \in\right.$ Aut $\left.\mathbb{Z}^{d}\right)$. Associated with each $\varphi$ in $\operatorname{Hom}\left(\Lambda^{2} \mathbb{Z}^{d}\right.$, $\mathbb{Z} / a \mathbb{Z})$ is a finite set of invariants completely describing the orbit of $\varphi$ under this action. The result holds with $\mathbb{Z}$ replaced by an arbitrary commutative principal ideal domain.
\end{abstract}

\section{NotATION}

In the following, $R$ denotes a commutative principal ideal domain (characteristic not equal to 2), $N$ the $R$-module $\bigoplus^{d} R=R^{d}$ with a fixed basis $\left\{e_{i} \mid i=1, \ldots, d\right\} \quad(d \in \mathbb{N})$, and $S$ a fixed complete set (containing 1) of nonassociates of $R$ ( $S$ may be chosen multiplicatively closed). If $A$ is a commutative ring, $m_{d}(A)$ denotes the ring of $d \times d$ matrices over $A$. If $a \in A$, the ideal $a A$ is written $(a)$ and $\pi$ denotes the canonical quotient map $A \rightarrow A /(a)$. If, in addition, $A$ is an $R$-algebra and $\varphi \in \operatorname{Hom}\left(\Lambda^{2} N, A\right)$, write $\varphi=\left[a_{1}, \ldots\right.$, $\left.a_{k}\right]$ if $k \leq d / 2,\left(a_{1}\right) \supseteq\left(a_{2}\right) \supseteq \cdots \supseteq\left(a_{k}\right) ; \varphi\left(e_{2 i-1} \wedge e_{2 i}\right)=a_{i} \quad(1 \leq i \leq k)$; and $\varphi\left(e_{j} \wedge e_{h}\right)=0$ for all other $j<h, j, h \in\{1, \ldots, d\}$.

\section{INTRODUCTION}

Consider $\Phi \in m_{d}(A), A$ a commutative principal ideal ring. Let $\left(d_{k}(\Phi)\right)$, the $k$ th determinantal divisor of $\Phi$, be the ideal of $A$ generated by the determinants of the $k \times k$ submatrices of $\Phi(1 \leq k \leq d)$. The greatest $k$ with $\left(d_{k}(\Phi)\right) \neq 0$ is the rank of $\Phi$. It is evident that $\left(d_{k}(\Phi)\right) \subseteq\left(d_{k-1}(\Phi)\right)$ where $\left(d_{0}(\Phi)\right)$ is defined to be $A$. For $k \geq 1$, let $\left(s_{k}(\Phi)\right)$ denote the ideal $\left\{m \in A \mid m d_{k-1}(\Phi) \in\left(d_{k}(\Phi)\right\}\right.$ if $\left(d_{k}(\Phi)\right) \neq 0$, and zero otherwise. Then $\left(s_{k}(\Phi)\right)\left(d_{k-1}(\Phi)\right)=\left(d_{k}(\Phi)\right)$. Note that $\left(s_{1}(\Phi)\right)=\left(d_{1}(\Phi)\right)$. Thus, for $\Phi_{1}$, $\Phi_{2} \in m_{d}(A),\left(s_{k}\left(\Phi_{1}\right)\right)=\left(s_{k}\left(\Phi_{2}\right)\right)$ for all $k$ if and only if $\left(d_{k}\left(\Phi_{1}\right)\right)=\left(d_{k}\left(\Phi_{2}\right)\right)$ for all $k$. If $A$ is also an integral domain then $\left(s_{k}(\Phi)\right) \subseteq\left(s_{k-1}(\Phi)\right)$ [3].

Received by the editors March 15, 1989 and, in revised form, April 24, 1989.

1980 Mathematics Subject Classification (1985 Revision). Primary 46K10, 46L40, 15A63, $46 \mathrm{~L} 80$.

Key words and phrases. Module, principal ideal domain, pfaffian, skew symmetric matrix, automorphism. 
Assume $A$ is both a commutative $R$-algebra and a principal ideal ring. The group $G=\operatorname{Aut}\left(R^{d}\right)$ acts on the $R$-module $\operatorname{Hom}\left(\Lambda^{2} R^{d}, A\right)$ by $\varphi \rightarrow \varphi(\alpha \wedge \alpha)$ $\left(\alpha \in \operatorname{Aut}\left(R^{d}\right)\right)$. Each $\varphi \in \operatorname{Hom}\left(\Lambda^{2} R^{d}, A\right)$ may be identified with a skew symmetric matrix $\Phi \in m_{d}(A)$ (the $(i, j)$ entry of $\Phi$ is $\left.\varphi\left(e_{i} \wedge e_{j}\right)\right)$. For each $\alpha \in \operatorname{Aut}\left(R^{d}\right)$, i.e., for each invertible matrix $\alpha$ in $m_{d}(R)$, the element of $m_{d}(A)$ associated with $\varphi(\alpha \wedge \alpha)$ is $\alpha^{t} \Phi \alpha$ (where $\alpha^{t}$ is the transpose of $\alpha)$. Define $\left(d_{k}(\varphi)\right)$ to be $\left(d_{k}(\Phi)\right)$ and $\left(s_{k}(\varphi)\right)$ to be $\left(s_{k}(\Phi)\right)$. It follows that $\left(d_{k}(\varphi(\alpha \wedge \alpha))\right)=\left(d_{k}(\varphi)\right)$ for all $k$ and thus $\left(s_{k}(\varphi(\alpha \wedge \alpha))\right)=\left(s_{k}(\varphi)\right)$ for all $k$ [3].

Recall that two elements $\Phi_{1}, \Phi_{2}$ of $m_{d}(A)$ are congruent if there is an $\alpha \in$ $\operatorname{Aut}\left(A^{d}\right)$ with $\Phi_{1}=\alpha^{t} \Phi_{2} \alpha$. They are equivalent if there are $\alpha, \beta \in \operatorname{Aut}\left(A^{d}\right)$ with $\Phi_{1}=\alpha \Phi_{2} \beta$. Thus, if $A=R$ the $G$-orbit of $\varphi$ in $\operatorname{Hom}\left(\Lambda^{2} R^{d}, R\right)$ is the same as the congruence class of the skew symmetric matrix $\Phi$. These congruence classes have been known for some time [3]. In this case, the rank $r$ of $\varphi$ is even, $\left(s_{2 k-1}(\varphi)\right)=\left(s_{2 k}(\varphi)\right)$ and the sequence of ideals $\left(s_{d}(\varphi)\right) \subseteq\left(s_{d-1}(\varphi)\right) \subseteq$ $\cdots \subseteq\left(s_{1}(\varphi)\right)$ is a complete invariant for the orbit of $\varphi$ in $\operatorname{Hom}\left(\Lambda^{2} R^{d}, R\right)$. Furthermore, there is an $\alpha \in \operatorname{Aut}\left(R^{d}\right)$ with

$$
\varphi(\alpha \wedge \alpha)=\left[s_{2}(\varphi), s_{4}(\varphi), \ldots, s_{r}(\varphi)\right]
$$

It follows from this that elements equivalent in $\operatorname{Hom}\left(\Lambda^{2} R^{d}, R\right)$ are also congruent [3].

The main new result below is a classification of the $G$-orbits in $\operatorname{Hom}\left(\Lambda^{2} R^{d}, A\right)$ if $A$ is the quotient, $R /(a)$, of $R$. An application is found in [1]. Although some special cases of the result (for example, when $R /(a)$ is a field, i.e., when $(a)$ is prime.) are easy consequences of the classification result for $A=R$, the general case does not seem to be.

For $\Phi \in m_{d}(\mathbb{Z} / a \mathbb{Z})$ complete invariants for the class $\{\alpha \Phi \beta \mid \alpha, \beta \in \operatorname{SL}(d, \mathbb{Z})\}$ are given in [2]. These conditions coincide with those given below for the $G$-orbit of $\varphi \in \operatorname{Hom}\left(\Lambda^{2} \mathbb{Z}^{d}, \mathbb{Z} /(a)\right)$. We conclude that if $\Phi_{1}, \Phi_{2}$ are skew symmetric elements of $m_{d}(\mathbb{Z} / a \mathbb{Z})$ with $\Phi_{1}=\alpha \Phi_{2} \beta$ for some $\alpha, \beta \in \operatorname{SL}(d, \mathbb{Z})$ then $\Phi_{1}=\gamma^{t} \Phi_{2} \gamma$ for some $\gamma \in \operatorname{SL}(d, \mathbb{Z})$.

Recall that if $A$ is a commutative ring and $d=2 m$, then there is a polynomial $P f$ in $d(d-1) / 2$ variables with coefficients in $A$ called the generic pfaffian (of size $d$ ). It is homogeneous of degree $m$. If $\varphi \in \operatorname{Hom}\left(\Lambda^{2} N, A\right)$ as before, write $\operatorname{Pf}(\varphi)$ for $P f(\Phi)$, the value of $P f$ evaluated at the elements $\varphi\left(e_{i} \wedge e_{j}\right) \quad(i<j)$ of $A$. Note that $P f(\varphi(\alpha \wedge \alpha))=\operatorname{Pf}\left(\alpha^{t} \Phi \alpha\right)=(\operatorname{det} \alpha) \operatorname{Pf}(\Phi)=$ $\operatorname{det} \alpha P f(\varphi)$ for $\alpha \in \operatorname{Aut}(N)$.

$$
\text { INVARIANTS OF } \varphi \in \operatorname{Hom}\left(\Lambda^{2} N, R /(a)\right)
$$

Choose $a \in R, a \neq 0$, and let $M$ denote the quotient $R /(a)$. In this section we describe invariants for the orbit of an element $\varphi \in \operatorname{Hom}\left(\Lambda^{2} N, M\right)$ under the action of $\operatorname{Aut}(N)$. 
For $\varphi \in \operatorname{Hom}\left(\Lambda^{2} N, M\right)$, let $L(\varphi)=\{n \in N \mid \varphi(n \wedge g)=0 \quad(g \in N)\}$. Since $N \cong a N \subseteq L(\varphi) \subseteq N, L(\varphi)$ is a free submodule of $N$ of rank $d$ and $I(\varphi)=N / L(\varphi)$ is a finitely generated torsion module over $R$. For $\alpha \in \operatorname{Aut}(N)$ we have $\alpha^{-1}(L(\varphi))=L(\varphi(\alpha \wedge \alpha))$; so $\alpha$ induces an isomorphism of $I(\varphi(\alpha \wedge \alpha))$ onto $I(\varphi)$. Thus the invariants of $I(\varphi)$, a sequence of decreasing ideals of $R$ completely describing the isomorphism class of $I(\varphi)$, are also invariants of the orbit of $\varphi$.

To compute the invariants of $I(\varphi)$, choose $\psi \in \operatorname{Hom}\left(\Lambda^{2} N, R\right)$ with $\pi \psi=$ $\varphi$. Since there is an $\alpha \in \operatorname{Aut}(N)$ with $\psi(\alpha \wedge \alpha)=\left[s_{2}(\psi), s_{4}(\psi), \ldots\right]$, it follows that $I(\varphi)$ has invariants $\left(p_{d}\right) \supseteq\left(p_{d-1}\right) \supseteq \cdots \supseteq\left(p_{1}\right)$, where $p_{j}$ are chosen with $p_{2 j-1}=p_{2 j}$ and $\left(p_{j}\right)\left(a, s_{j}(\psi)\right)=(a)$. (Define $\left(p_{r+1}\right)=\cdots=\left(p_{d}\right)=(1)$ if the number of invariants of $I(\varphi)$ is $r$ and $r$ is less than $d$.) Since $(a) \subseteq\left(p_{j}\right)$ for all $j$, the ideals $\left(p_{j}\right)$ of $R$ may be viewed equally well as ideals of $M$. However, the ideals $\left(p_{j}\right)$ have little relationship to the ideals $\left(s_{j}(\varphi)\right)$ of $M$. Although $\pi\left(d_{j}(\psi)\right)=d_{j}(\varphi)$, we have $\pi\left(s_{j}(\psi)\right) \subseteq\left(s_{j}(\varphi)\right)$ unless $\left(d_{j}(\varphi)\right)=0$, in which case $0=\left(s_{j}(\varphi)\right) \subseteq \pi\left(s_{j}(\psi)\right)$.

Definition. For $\varphi \in \operatorname{Hom}\left(\Lambda^{2} N, M\right)$ and $d=2 m$, let

$$
C(\varphi)=\pi\left(P f(\psi) \delta_{1}^{-1} \delta_{2}^{-1} \cdots \delta_{m-1}^{-1}\right) .
$$

Here $\psi \in \operatorname{Hom}\left(\Lambda^{2} N, R\right)$ satisfies $\pi(\psi)=\varphi$, and $\delta_{j} \in S$ is such that $\left(\delta_{j}\right)=$ $\left(s_{2 j}(\psi), a\right), j=1, \ldots, m$.

Of course, we must show that $C(\varphi)$ is well defined. Note first that the elements $\delta_{j}, j=1, \ldots, m$, are uniquely determined by $\varphi$. This follows from the fact that the ideals $\left(a, s_{j}(\psi)\right)$ of $R$ are uniquely determined by the conditions $\left(p_{j}\right)\left(a, s_{j}(\psi)\right)=(a)$, where $\left(p_{j}\right)$ are the invariants of $I(\varphi)$. Therefore, $\left(a, s_{j}(\psi)\right)=\left(a, s_{j}\left(\psi^{\prime}\right)\right)$ for $\psi, \psi^{\prime} \in \operatorname{Hom}\left(\Lambda^{2} N, R\right)$, with $\pi \psi=\pi \psi^{\prime}=\varphi$. Choose $\alpha \in \operatorname{Aut}(N)$ with $\psi(\alpha \wedge \alpha)=\left[s_{2}(\psi), s_{4}(\psi), \ldots, s_{2 m}(\psi)\right]$. We have

$$
\begin{aligned}
s_{2}(\psi) \ldots s_{2 m}(\psi) \delta_{1}^{-1} \cdots \delta_{m-1}^{-1} & =P f(\psi(\alpha \wedge \alpha)) \delta_{1}^{-1} \cdots \delta_{m-1}^{-1} \\
& =\mu P f(\psi) \delta_{1}^{-1} \cdots \delta_{m-1}^{-1},
\end{aligned}
$$

where $\mu=\operatorname{det} \alpha$ is a unit of $R$. This also demonstrates that $\delta_{1} \cdots \delta_{m-1}$ divides $P f(\psi)$. Since $\psi^{\prime}(\alpha \wedge \alpha)=\psi(\alpha \wedge \alpha) \bmod a, \psi^{\prime}(\alpha \wedge \alpha)$ has entries in $(a)$ or $s_{j}(\psi)+(a)$. Also, $\delta_{j} \mid s_{k}(\psi) \quad(k \geq 2 j)$ and $\delta_{j} \mid a$. It follows from the form of the polynomial $P f$ that

$$
\begin{aligned}
\mu P f\left(\psi^{\prime}\right) \delta_{1}^{-1} \cdots \delta_{m-1}^{-1} & =P f\left(\psi^{\prime}(\alpha \wedge \alpha)\right) \delta_{1}^{-1} \cdots \delta_{m-1}^{-1} \\
& =\prod_{j=1}^{m}\left(s_{2 j}(\psi)+x_{j} a\right) \delta_{1}^{-1} \cdots \delta_{m-1}^{-1}+\sum_{t} g_{t} \delta_{1}^{-1} \cdots \delta_{m-1}^{-1},
\end{aligned}
$$

where $x_{j} \in R$ and $g_{t} \in R$ is divisible by $\delta_{1} \cdots \delta_{m-1}$ with $g_{t} \delta_{1}^{-1} \cdots \delta_{m-1}^{-1} \in(a)$. 
The first term of the sum expands to

$$
\prod_{j=1}^{m} s_{2 j}(\psi) \delta_{1}^{-1} \cdots \delta_{m-1}^{-1}+a \sum_{q} f_{q} \delta_{1}^{-1} \cdots \delta_{m-1}^{-1}
$$

with $f_{q} \in R$ divisible by $\delta_{1} \cdots \delta_{m-1}$. Therefore,

$$
\mu P f\left(\psi^{\prime}\right) \delta_{1}^{-1} \cdots \delta_{m-1}^{-1}=\mu P f(\psi) \delta_{1}^{-1} \cdots \delta_{m-1}^{-1}+x a
$$

for some $x \in R$, and

$$
\pi\left(P f\left(\psi^{\prime}\right)\left(\delta_{1}^{-1} \cdots \delta_{m-1}^{-1}\right)=\pi\left(P f(\psi) \delta_{1}^{-1} \cdots \delta_{m-1}^{-1}\right) .\right.
$$

Proposition. If $d=2 m, \varphi \in \operatorname{Hom}\left(\Lambda^{2} N, M\right)$, and $\alpha \in \operatorname{Aut}(N)$, then

$$
C(\varphi(\alpha \wedge \alpha))=\pi(\operatorname{det} \alpha) C(\varphi) .
$$

Proof. Choose $\psi \in \operatorname{Hom}\left(\Lambda^{2} N, R\right)$ with $\pi \psi=\varphi$ and $\delta_{j} \in S$ with $\left(\delta_{j}\right)=$ $\left(s_{2 j}(\psi), a\right), j=1, \ldots, m$. Since $I(\varphi(\alpha \wedge \alpha))=I(\varphi)$, it follows that

$$
\left.s_{2 j}(\psi(\alpha \wedge \alpha)), a\right)=\left(s_{2 j}(\psi), a\right) \text {. }
$$

We have

$$
\begin{aligned}
C(\varphi(\alpha \wedge \alpha)) & =\pi\left(P f(\psi(\alpha \wedge \alpha)) \delta_{1}^{-1} \cdots \delta_{m-1}^{-1}\right) \\
& =\pi\left(\operatorname{det}(\alpha) P f(\psi) \delta_{1}^{-1} \cdots \delta_{m-1}^{-1}\right)=\pi(\operatorname{det} \alpha) C(\varphi) .
\end{aligned}
$$

Note that $C(\varphi)=0$ whenever $\left(\delta_{j}\right)=(a)$ for some $j$. Note also that $C(\varphi)$ is a finer invariant than $P f(\varphi)$. For example, let $R$ be the ring of integers, $d=4$ and $a=27$. If $\varphi_{1}=[\pi(3), \pi(6)]$ and $\varphi_{2}=[\pi(3), \pi(24)]$, then $I\left(\varphi_{1}\right)=I\left(\varphi_{2}\right)$ and $P f\left(\varphi_{1}\right)=P f\left(\varphi_{2}\right)=\pi(18)$. However, $C\left(\varphi_{1}\right)=\pi(6)$ and $C\left(\varphi_{2}\right)=\pi(24)$; so it follows from the result below that $\varphi_{1}$ and $\varphi_{2}$ are not in the same orbit under the action of $\operatorname{Aut}\left(\mathbb{Z}^{4}\right)$. These elements are congruent though, since there is an invertible $\alpha \in m_{4}(\mathbb{Z} / 27)$ with $\alpha^{t} \varphi_{1} \alpha=\varphi_{2}$.

\section{Classification OF ORBITS}

Theorem 1. Let $R$ be a principal ideal domain, $N=\bigoplus^{d} R, M=R /(a) a$ quotient of $R, \mu$ a unit of $R$, and $\varphi_{1}, \varphi_{2} \in \operatorname{Hom}\left(\Lambda^{2} N, M\right)$.

(i) Assume $d$ is odd. If there is an $\alpha \in \operatorname{Aut}(N)$ with $\varphi_{1}(\alpha \wedge \alpha) \cong \varphi_{2}$, then $I\left(\varphi_{1}\right) \cong I\left(\varphi_{2}\right)$. Conversely, if $I\left(\varphi_{1}\right) \cong I\left(\varphi_{2}\right)$, then there is an $\alpha \in \operatorname{Aut}(N)$ with $\operatorname{det} \alpha=\mu$ and $\varphi_{1}(\alpha \wedge \alpha)=\varphi_{2}$.

(ii) Assume $d$ is even. There is an $\alpha \in \operatorname{Aut}(N)$ with $\operatorname{det} \alpha=\mu$ and $\varphi_{1}(\alpha \wedge \alpha)$ $=\varphi_{2}$ if and only if $I\left(\varphi_{1}\right)=I\left(\varphi_{2}\right)$ and $C\left(\varphi_{2}\right)=\pi(\mu) C\left(\varphi_{1}\right)$.

If $R /(a)$ is an integral domain, i.e., a field, then this theorem is easy. (Use the canonical form of a skew symmetric matrix over a field and the fact that for any $a \in R$, an element of $\operatorname{SL}(d, R /(a))$ can be lifted to an element of $\mathrm{SL}(d, R)[3]$. 
We first prove two Lemmas.

Lemma 1 (Use the preceding notation). Let $d=3, \varphi_{1}=[\pi(u)], \varphi_{2}=[\pi(v)]$, and $(u, a)=(v, a)=(\delta)$, where $u, v, \delta \in R$. There is an $\alpha \in \operatorname{Aut}(N)$ with $\operatorname{det} \alpha=\mu$ and $\varphi_{1}(\alpha \wedge \alpha)=\varphi_{2}$.

Proof. Choose $x, y \in R$ with $x u+y a=\delta$ and define $\varphi \in \operatorname{Hom}\left(\Lambda^{2} N, M\right)$ by $\varphi=[\pi(\delta)]$. Using the fact that $\pi\left(a \delta^{-1}\right) \pi(u)=\pi(a) \pi\left(u \delta^{-1}\right)=0$ we have $\varphi_{1}(\gamma \wedge \gamma)=\varphi$, where $\gamma \in \operatorname{Aut}(N)$ is defined by $\gamma\left(e_{1}\right)=x e_{1}+y e_{3}$, $\gamma\left(e_{2}\right)=e_{2}, \gamma\left(e_{3}\right)=-\mu a \delta^{-1} e_{1}+\mu u \delta^{-1} e_{3}$. Note that $\operatorname{det} \gamma=\mu$. Similarly there is $\beta \in \operatorname{Aut}(N)$, with $\operatorname{det} \beta=1$ and $\varphi_{2}(\beta \wedge \beta)=\varphi$. Let $\alpha=\gamma \beta^{-1}$.

Lemma 2 (Use the preceding notation). Let $d=4, \varphi_{1}=\left[\pi\left(u_{1}\right), \pi\left(u_{2}\right)\right]$, $\varphi_{2}=\left[\pi\left(v_{1}\right), \pi\left(v_{2}\right)\right]$, and $\left(\pi\left(u_{i}\right)\right)=\left(\pi\left(v_{i}\right)\right)$, where $u_{i}, v_{i} \in R$ for $i=1,2$. Assume $\pi(\mu) C\left(\varphi_{1}\right)=C\left(\varphi_{2}\right)$. Then there is an $\alpha \in \operatorname{Aut}(N)$ with $\operatorname{det} \alpha=\mu$ and $\varphi_{1}(\alpha \wedge \alpha)=\varphi_{2}$.

Proof. Choose $\delta_{i} \in S$ with $\left(\delta_{i}\right)=\left(u_{i}, a\right)=\pi^{-1}\left(\pi\left(u_{i}\right)\right), i=1,2$. Then $\pi\left(\mu u_{1} u_{2} \delta_{1}^{-1}\right)=\pi(\mu) C\left(\varphi_{1}\right)=C\left(\varphi_{2}\right)=\pi\left(v_{1} v_{2} \delta_{1}^{-1}\right)$. There are $x, y \in R$ with $x u_{1}+y a=\delta_{1}$. Since $\left(v_{1} \delta_{1}^{-1}, a \delta_{1}^{-1}\right)=(1)=\left(x, a \delta_{1}^{-1}\right)$ and since $R$ is a unique factorization domain, we have $\left(z,\left(a \delta_{1}^{-1}\right)^{2}\right)=\left(z, a \delta_{1}^{-1}\right)=(1)$, where $z=v_{1} \delta_{1}^{-1} x$. Thus there are $p, q \in R$ with $p z+q\left(a \delta_{1}^{-1}\right)^{2}=1$. Define $\alpha \in \operatorname{Aut}(N)$ with $\operatorname{det} \alpha=\mu$ by $\alpha\left(e_{1}\right)=z e_{1}+a \delta_{1}^{-1} e_{3}, \alpha\left(e_{2}\right)=e_{2}$, $\alpha\left(e_{3}\right)=-q a \delta_{1}^{-1} e_{1}+p e_{3}$, and $\alpha\left(e_{4}\right)=\mu e_{4}$.

Since $\pi(z) \pi\left(u_{1}\right)=\pi\left(v_{1} \delta_{1}^{-1}\right) \pi\left(\delta_{1}-y a\right)=\pi\left(v_{1}\right)$, we have $\varphi_{1}(\alpha \wedge \alpha)\left(e_{1} \wedge e_{2}\right)=$ $\varphi_{2}\left(e_{1} \wedge e_{2}\right)$. Note that

and

$$
\begin{aligned}
\pi\left(p z v_{2}\right) & =\pi\left(1-q\left(a \delta_{1}^{-1}\right)^{2} \pi\left(v_{2}\right)\right. \\
& =\pi\left(v_{2}\right)-\pi\left(q\left(a \delta_{1}^{-1}\right)\left(v_{2} \delta_{1}^{-1}\right) a\right) \\
& =\pi\left(v_{2}\right)
\end{aligned}
$$

$$
\pi\left(p z v_{2}\right)=\pi\left(p x v_{1} v_{2} \delta_{1}^{-1}\right) .
$$

By assumption this is $\pi\left(p x \mu u_{1} u_{2} \delta_{1}^{-1}\right)$ which is

$$
\begin{aligned}
\pi\left(p \mu u_{2}\left(u_{1} \delta_{1}^{-1}\right) x\right) & =\pi\left(p \mu u_{2}\left(1-y a \delta_{1}^{-1}\right)\right) \\
& =\pi\left(p \mu u_{2}\right)-\pi\left(p \mu\left(u_{2} \delta_{1}^{-1}\right) y a\right) \\
& =\pi\left(p \mu u_{2}\right) .
\end{aligned}
$$

Thus, $\pi\left(v_{2}\right)=\pi\left(p \mu u_{2}\right)$ and $\varphi_{1}(\alpha \wedge \alpha)\left(e_{3} \wedge e_{4}\right)=\varphi_{2}\left(e_{3} \wedge e_{4}\right)$. It is straightforward to complete the check that $\varphi_{1}(\alpha \wedge \alpha)=\varphi_{2}$.

The following fact is needed in the proof of Theorem 1(ii). If $\sigma, \rho \in R$ with $(\pi(\sigma))=(\pi(\rho))$ (equivalently, $(\sigma, a)=(\rho, a))$, then there is a $w \in R$ with $\pi(w \sigma)=\pi(\rho)$ and $\pi(w)$ a unit in $R /(a)$. There is a straightforward proof of this using the Chinese Remainder theorem. 
Proof of Theorem 1. (i) Let $d=2 m+1$. Choose $\psi_{j} \in \operatorname{Hom}\left(\Lambda^{2} N, R\right)$ with $\pi \psi_{j}=\varphi_{j}, j=1,2$. Assuming $I\left(\varphi_{1}\right) \cong I\left(\varphi_{2}\right)$, we have $\left(a, s_{k}\left(\psi_{1}\right)\right)=$ $\left(a, s_{k}\left(\psi_{2}\right)\right)$ for $k=1, \ldots, d$. There are $\beta_{j} \in \operatorname{Aut}(N)$ with $\varphi_{j}\left(\beta_{j} \wedge \beta_{j}\right)=$ $\left[\pi\left(s_{2}\left(\psi_{j}\right), \pi\left(s_{4}\left(\psi_{j}\right)\right), \ldots, \pi\left(s_{2 m}\left(\psi_{j}\right)\right)\right]\right.$. Let $N_{1}$ denote the submodule of $N$ spanned by $\left\{e_{1}, e_{2}, e_{d}\right\}, N_{r}$ the submodule spanned by the remaining $e_{i}$, and $I_{r}$ the identity map of $N_{r}$. Since $\Lambda^{2} N=\Lambda^{2} N_{1} \oplus N_{1} \otimes N_{r} \oplus \Lambda^{2} N_{r}$, Lemma 1 ensures the existence of an $\alpha_{1} \in \operatorname{Aut}(N)$ (of the form $\gamma_{1} \oplus I_{r}$ ) with $\operatorname{det}\left(\alpha_{1}\right)$ any prescribed unit of $R$ and

$$
\varphi_{1}\left(\beta_{1} \wedge \beta_{1}\right)\left(\alpha_{1} \wedge \alpha_{1}\right)=\left[\left(\pi\left(s_{2}\left(\psi_{2}\right)\right), \pi\left(s_{4}\left(\psi_{1}\right), \ldots\right] .\right.\right.
$$

Repeating this argument yields $\alpha_{k} \in \operatorname{Aut}(N), k=1, \ldots, m$, with

$$
\varphi_{1}\left(\beta_{1} \wedge \beta_{1}\right)\left(\alpha_{1} \wedge \alpha_{1}\right) \cdots\left(\alpha_{k} \wedge \alpha_{k}\right)=\varphi_{2}\left(\beta_{2} \wedge \beta_{2}\right) \text {. }
$$

By choosing $\operatorname{det} \alpha_{k}$ appropriately and setting $\alpha=\beta_{1} \alpha_{1} \cdots \alpha_{m} \beta_{2}^{-1}$, we have $\operatorname{det} \alpha=\mu$ and $\varphi_{1}(\alpha \wedge \alpha)=\varphi_{2}$.

(ii) Let $d=2 m$ and assume $I\left(\varphi_{1}\right) \cong I\left(\varphi_{2}\right)$ and $C\left(\varphi_{2}\right)=\pi(\mu) C\left(\varphi_{1}\right)$. If $\psi_{j} \in \operatorname{Hom}\left(\Lambda^{2} N, R\right)$ are chosen with $\pi \psi_{j}=\varphi_{j}, j=1,2$, there are $\delta_{i} \in S$ with $\left(\delta_{i}\right)=\left(a, \sigma_{i}\right)=\left(a, \rho_{i}\right)$, where $\sigma_{i}$ and $\rho_{i}$ denote $s_{2 i}\left(\psi_{1}\right)$ and $s_{2 i}\left(\psi_{2}\right)$ respectively, $i=1, \ldots, m$. Choose $\beta_{1}, \beta_{2} \in \operatorname{Aut}(N)$ such that $\varphi_{1}\left(\beta_{1} \wedge \beta_{1}\right)=$ $\left[\pi\left(\sigma_{1}\right), \pi\left(\sigma_{2}\right), \ldots, \pi\left(\sigma_{m}\right)\right]$ and $\varphi_{2}\left(\beta_{2} \wedge \beta_{2}\right)=\left[\pi\left(\rho_{1}\right), \pi\left(\rho_{2}\right), \ldots, \pi\left(\rho_{m}\right)\right]$. The condition $C\left(\varphi_{2}\right)=\pi(\mu) C\left(\varphi_{1}\right)$ is equivalent to

$$
\pi\left(\rho_{1} \cdots \rho_{m} \delta_{1}^{-1} \cdots \delta_{m-1}^{-1}\right)=\pi\left(\mu_{2} \mu \mu_{1}^{-1}\right) \pi\left(\sigma_{1} \cdots \sigma_{m} \delta_{1}^{-1} \cdots \delta_{m-1}^{-1}\right),
$$

where $\mu_{j}=\operatorname{det} \beta_{j}, j=1,2$.

If $m=1$, any $\gamma \in \operatorname{Aut}(N)$ with $\operatorname{det} \gamma=\mu_{2} \mu \mu_{1}^{-1}$ satisfies $\varphi_{1}\left(\beta_{1} \wedge \beta_{1}\right)(\gamma \wedge \gamma)=$ $\varphi_{2}\left(\beta_{2} \wedge \beta_{2}\right)$. If $\alpha=\beta_{1} \gamma \beta_{2}^{-1}$, it follows that $\operatorname{det} \alpha=\mu$ and $\varphi_{1}(\alpha \wedge \alpha)=\varphi_{2}$. Henceforth assume $m>1$. Then

Let $x_{i}, y_{i} \in R$ with $\rho_{i} x_{i}+a y_{i}=\delta_{i}$ and set $u_{i}=\sigma_{i} \delta_{i}^{-1} x_{i}, i=1, \ldots, m-1$.

$$
\pi\left(u_{i} \rho_{i}\right)=\pi\left(\sigma_{i} \delta_{i}^{-1}\left(\delta_{i}-a y_{i}\right)\right)=\pi\left(\sigma_{i}\right) .
$$

Also choose $w_{i}, v_{i} \in R$ with $\pi\left(w_{i} \rho_{i}\right)=\pi\left(\sigma_{i}\right)$ and $\pi\left(w_{i} v_{i}\right)=\pi(1), i=1$, $\ldots, m-1$. Then $\pi\left(v_{i} \sigma_{i}\right)=\pi\left(\rho_{i}\right)$ and

$$
\pi\left(v_{i} u_{i} \rho_{m-1}\right)=\pi\left(v_{i} u_{i} \rho_{i}\left(\rho_{i}^{-1} \rho_{m-1}\right)\right)=\pi\left(\rho_{i} \rho_{i}^{-1} \rho_{m-1}\right)=\pi\left(\rho_{m-1}\right)
$$

for $i \leq m-1$.

For $i \leq k \leq m-1$ define $\xi_{k} \in \operatorname{Hom}\left(\Lambda^{2} N, M\right)$ as

$$
\left[\pi\left(\rho_{1}\right), \ldots, \pi\left(\rho_{k-1}\right), \pi\left(w_{1} \cdots w_{k-1} \sigma_{k}\right), \pi\left(\sigma_{k+1}\right), \ldots, \pi\left(\sigma_{m}\right)\right] .
$$

Note that $\xi_{1}=\varphi_{1}\left(\beta_{1} \wedge \beta_{1}\right)$.

Since $\pi\left(w_{1} \cdots w_{k-1} \sigma_{k} \sigma_{k+1} \delta_{k}^{-1}\right)=\pi\left(\rho_{k} w_{1} \cdots w_{k} \sigma_{k+1} \delta_{k}^{-1}\right)$ for $1 \leq k \leq m-2$, an application of Lemma 2 (recall that $\pi\left(w_{i}\right)$ are units of $M$ ) yields an $\alpha_{k} \in$ $\operatorname{Aut}(N)$ with $\operatorname{det} \alpha_{k}=1$ and $\xi_{k}\left(\alpha_{k} \wedge \alpha_{k}\right)=\xi_{k+1}(1 \leq k \leq m-2)$. 
We claim that

$$
\pi\left(\mu_{1}^{-1} \mu \mu_{2}\right) \pi\left(w_{1} \cdots w_{m-2} \sigma_{m-1} \sigma_{m} \delta_{m-1}^{-1}\right)=\pi\left(\rho_{m-1} \rho_{m} \delta_{m-1}^{-1}\right),
$$

from which it follows that there is an $\alpha_{m-1} \in \operatorname{Aut}(N)$ with $\operatorname{det} \alpha_{m-1}=\mu_{1}^{-1} \mu \mu_{2}$ and $\xi_{m-1}\left(\alpha_{m-1} \wedge \alpha_{m-1}\right)=\varphi_{2}\left(\beta_{2} \wedge \beta_{2}\right)$. We have

$$
\begin{aligned}
\pi\left(\mu_{1}^{-1}\right. & \left.\mu \mu_{2} \delta_{m-1}^{-1} \sigma_{m}\left(w_{1} \cdots w_{m-2} \sigma_{m-1}\right)\right) \\
& =\pi\left(\mu_{1}^{-1} \mu \mu_{2} \delta_{m-1}^{-1} \sigma_{m}\left(w_{1} \cdots w_{m-2} u_{m-1} \rho_{m-1}\right)\right) \\
& =\pi\left(\mu_{1}^{-1} \mu \mu_{2} \delta_{m-1}^{-1} \sigma_{m}\left(w_{1} \cdots w_{m-2}\left(v_{1} u_{1} v_{2} u_{2} \cdots v_{m-2} u_{m-2} u_{m-1} \rho_{m-1}\right)\right)\right) \\
& =\pi\left(\mu_{1}^{-1} \mu \mu_{2} \delta_{m-1}^{-1} \rho_{m-1}\left(w_{1} v_{1}\right)\left(w_{2} v_{2}\right) \cdots\left(w_{m-2} v_{m-2}\right) u_{1} \cdots u_{m-1} \sigma_{m}\right) \\
& =\pi\left(\mu_{1}^{-1} \mu \mu_{2} \delta_{m-1}^{-1} \rho_{m-1} \sigma_{1} \cdots \sigma_{m-1} x_{1} \cdots x_{m-1} \delta_{1}^{-1} \cdots \delta_{m-1}^{-1} \sigma_{m}\right) \\
& =\pi\left(\delta_{m-1}^{-1} \rho_{m-1} \rho_{1} \cdots \rho_{m} \delta_{1}^{-1} \cdots \delta_{m-1}^{-1} x_{1} \cdots x_{m-1}\right) \\
& =\pi\left(\delta_{m-1}^{-1} \rho_{m-1}\left(\rho_{1} \delta_{1}^{-1} x_{1}\right) \cdots\left(\rho_{m-1} \delta_{m-1}^{-1} x_{m-1}\right) \rho_{m}\right) \\
& =\pi\left(\delta_{m-1}^{-1} \rho_{m-1} \rho_{m}\right) .
\end{aligned}
$$

The last equality follows from

$$
\pi\left(\rho_{i} \delta_{i}^{-1} x_{i} \rho_{m}\right)=\pi\left(\left(1-a \delta_{i}^{-1} y_{i}\right) \rho_{m}\right)=\pi\left(\rho_{m}\right)-\pi\left(\rho_{m} \delta_{i}^{-1} y_{i} a\right)=\pi\left(\rho_{m}\right) .
$$

Letting $\alpha=\beta_{1} \alpha_{1} \cdots \alpha_{m-1} \beta_{2}^{-1}$, we have $\operatorname{det} \alpha=\mu$ and $\varphi_{1}(\alpha \wedge \alpha)=\varphi_{2}$.

Thus, if $d$ is odd, the orbits in $\operatorname{Hom}\left(\Lambda^{2} N, M\right)$ are the same for the action of $\operatorname{Aut}(N)$ and the action of the subgroup $\operatorname{SL}(d, R)$. Since an element of $\operatorname{SL}(d, M)$ can be lifted to an element of $\operatorname{SL}(d, R)$, we also know the orbit structure for the action of $\operatorname{SL}(d, M)$. If $d$ is even, the orbit structure is now also clear for the action of $\operatorname{SL}(d, R)$ (and $\operatorname{SL}(d, M))$.

\section{REFERENCES}

1. B. Brenken, $A$ classification of some noncommutative tori (presented at the Great Plains Operator Theory Seminar, May, 1987 in Lawrence, Kansas, (to appear).

2. F. G. Frobenius, Theorie der linearen Formen mit ganzen coefficienten, J. Reine Angew. Math. 86 (1880), 96-116.

3. M. Newman, Integral matrices, Academic Press, 1972.

Mathematics Department, University of Calgary, Calgary, Alberta, T2N in4, CANADA 